\section{Cahiers de Narratologie}

Analyse et théorie narratives

$10.1 \mid 2001$

La voix narrative

\title{
Oscillation et dédoublement des voix narratives dans El Alhajadito (1961) de Miguel Angel
}

« Estaba en su corredorcito. Nada era real. Imaginación. Sueños. Cuentos de las criadas viejas*»

\section{Dante Barrientos Tecùn}

\section{(2) OpenEdition}

\section{Journals}

Electronic version

URL: http://journals.openedition.org/narratologie/6924

DOI: 10.4000/narratologie.6924

ISSN: 1765-307X

Publisher

LIRCES

\section{Printed version}

Date of publication: 1 January 2001

Number of pages: 123-136

ISBN: 2914561032

ISSN: 0993-8516

Electronic reference

Dante Barrientos Tecùn, "Oscillation et dédoublement des voix narratives dans El Alhajadito (1961) de Miguel Angel", Cahiers de Narratologie [Online], 10.1 | 2001, Online since 20 October 2014, connection on 24 February 2021. URL: http://journals.openedition.org/narratologie/6924 ; DOI: https://doi.org/ 10.4000/narratologie.6924 


\title{
OSCILLATION ET DÉDOUBLEMENT DES VOIX NARRATIVES DANS \\ EL ALHAJADITO (1961) DE MIGUEL ANGEL ASTURIAS :
}

«Estaba en su corredorcito. Nada era real. Imaginación.

Sueños. Cuentos de las criadas viejas. ${ }^{1}$

\author{
Dante BARRIENTOS TECÚN \\ Université d'Aix-en-Provence
}

On sait maintenant que la plupart des textes narratifs de Miguel Angel Asturias (1899-1974) ont connu une histoire singulière. Elaborés et racontés oralement avant même d'être écris - tel le cas des Leyendas de Guatemala (1930) et de El Señor Presidente (1946) -, ou soumis à une attente de publication forcée, le temps qu'une dictature soit renversée ; ou encore qu'ils soient écris dans les circonstances d'une invasion contre son pays - le cas de la « Trilogie »-, le fait est que les textes narratifs de notre auteur connaissent une série d'avatars dans le processus d'écriture - publication qui répercute tant sur leur architecture que sur le regard polémique du discours critique ${ }^{2}$.

De ces histoires singulières l'une d'entre elles nous semble particulièrement significative à cause de son influence sur l'assemblage du texte, c'est l'histoire du processus d'écriture de El Alhajadito (1961). Ce roman - d'après les études

1 Miguel Angel ASTURIAS, El Alhajadito, Buenos Aires, Losada, 1966, $161 \mathrm{pp}$. Sexta edición. Biblioteca clásica y contemporánea. Citation p. 12. Nous avons travaillé avec cette édition. Pour les citations postérieures, nous indiquerons uniquement la page après l'extrait.

2 C'est le cas de la "Trilogie»-Viento Fuerte (1949), El Papa Verde (1954), Los ojos de los enterrados (1960) - et de Week-End en Guatemala (1956), ouvrages jugés souvent comme des textes trop marqués par le signe idéologique. 
plus récentes ${ }^{3}$ - a été commencé durant la période parisienne d'Asturias, entre 1925 et 1928 , et lorsque le romancier retourne au Guatemala (1933), il ne semble pas avoir repris sa rédaction. Davantage, car l'auteur avait même oublié l'existence du manuscrit. Ce n'est que vers le début des années 50, la Révolution d'octobre 1944 ayant triomphé au Guatemala et Asturias étant à l'époque nommé Ambassadeur au Salvador par le gouvernement de Jacobo Arbenz, qu'il tombe, par hasard, sur le manuscrit ${ }^{4}$. Un processus de réécriture est entamé, ce qui mènera l'auteur à envisager la possibilité de fusionner ce texte avec un autre roman, Maladrón (1969), et de faire ainsi des deux textes un seul livres. Nous avons donc là un cas très singulier d'un roman à écriture discontinue, résultat d'une rédaction entreprise à des époques différentes et de projets créatifs en évolution.

3 Voir dans la Collection Archivos, Catálogo La riqueza de la diversidad, Vida, obra y herencia de Miguel Angel Asturias 1899 - 1999, Paris, ALLCA XX / Ediciones Unesco, 1999, 688 pp., en particulier Jean-Philippe Barnabé, "En el principio existía la leyenda », p. 510 et Aline Janquart, "El manuscrito de las sorpresas », p. 562. Voir aussi Giuseppe Bellini, «El regreso al misterioso mundo de la magia, El Alhajadito y Mulata de tal», p. 127-128, dans son ouvrage Mundo mágico y mundo real. La narrativa de Miguel Angel Asturias, Roma, Bulzoni Editores, 1999, 242 pp.

4 D'après son propre témoignage, au moment du déménagement à San Salvador, son épouse retourne à la capitale guatémaltèque pour organiser le transport de livres et d'autres objets. Entre des papiers partiellement brûlés se trouve le manuscrit de El Alhajadito, lui aussi en partie brûlé : «Entonces, entre papeles quemados y trastos viejos, encontró casi íntegro el manuscrito de El Ahijadito (sic), o, mejor dicho, de la novela que llevó más tarde ese nombre al ser publicada en Buenos Aires. Estaba casi entera, pero hube de reescribir lo que faltaba por haber sido quemado. Y si se salvó el manuscrito, y si se salvaron ciertos poemas, se lo debo a la vieja Lola Reyes, quien me había cuidado de niño. Lola Reyes es la Tomasa de mi obra teatral Soluna. El lenguaje de Tomasa es el de Lola Reyes. Hubo en casa de mi madre un fuego que Lola Reyes se lanzó a apagar con una frazada en la mano. "Luis López Alvarez, Conversaciones con Miguel Angel Asturias, Madrid, Editorial Magisterio Español, 1974, 215 pp. Citation p. 197-198.

5 Voir dans la Collection Archivos, Aline Janquart, op. cit., p. 562. 
Si je me suis quelque peu attardé sur l'histoire du texte, c'est parce que les circonstances d'écriture de El Alhajadito ont produit un assemblage du roman qui peut sembler " décousu » 6 , fragmenté en voix narratives très hétérogènes. Or c'est cette hétérogénéité qui, à notre sens, comporte l'une des clés du roman et laisse entrevoir la nature du projet esthétique d'Asturias. Dans ce travail, nous nous proposons donc de mettre en avant comment Asturias parvient à entrelacer une multiplicité de voix dont le modèle est constitué par la tradition populaire orale.

$* * *$

La construction des espaces narratifs du roman se fait à partir d'un enchaînement de voix : celles des ancêtres du protagoniste, de son monde populaire, de la tradition orale. Mais cette pluralité se concentre en une seule voix, celle du "Alhajadito ». Cette voix unique et multiple à la fois - nous l'avançons maintenant - n'est autre chose que la représentation, dans le texte, de l'inconscient culturel. Ainsi, dans le roman, il s'agira d'espaces à double dimension : celle de l'histoire du protagoniste et celle de son imaginaire, du monde des rêves. Le lecteur est conduit abruptement d'une dimension à l'autre, dans un mouvement oscillatoire, ce qui provoque une sensation de structure dispersée.

Du point de vue de la structure externe, le roman est organisé en trois parties ${ }^{7}$ qui se centrent autour d'un espace

6 Jean Philippe BARNABÉ écrit : «el examen de los manuscritos, es decir la perspectiva de la escritura, nos lleva a proponer una lectura desarticulada, por así decirlo, de El Alhajadito, libro que resulta de un ensamblaje tardío y algo forzado de tres elementos marcadamente dispares, por haber sido concebidos en momentos y con propósitos distintos, como lo demuestra el cuaderno $\mathrm{n}^{\circ} 5$, que contiene una primera versión, titulada " $\mathrm{ki}$, el barco líquido », de la segunda parte, redactada mucho antes que todo lo demás, y de manera enteramente autónoma »op. cit., p. 510.

7 Chacune des parties est divisée en chapitres, sans titres. La première partie comporte 21 chapitres, la deuxième ne comporte qu'un seul long chapitre, une vingtaine de pages environ, et la troisième compte huit chapitres. 
principal : un petit couloir - « el corredorcito »-(1ère Partie $)^{8}$, un bateau fantôme (2ème Partie), une maison familiale (3ème Partie). Si des voix narratives différentes occupent et construisent ces univers, on peut établir une corrélation plus étroite entre la première et la troisième parties. En effet, dans ces deux parties la narration est organisée en fonction d'une même voix qui se dédouble par rapport à "l'Alhajadito ", d'abord en tant qu'enfant et ensuite en tant qu'adulte. Quant à la deuxième partie, il s'agit d'un rêve qui a lieu sur un bateau fantôme et qui prend sa source dans les histoires magiques que le protagoniste écoute et réinvente à son tour9 .

Le roman s'ouvre avec la présence du «Alhajadito » dans le «petit couloir». Personnage énigmatique dans la mesure où des repères précis sur sa vie ou son apparence physique sont absents. On doit se contenter de savoir qu'il s'agit d'un descendant d'une mystérieuse lignée de « Alhajados » (" parés de joyaux ») disparus, mais qui, grâce à la mémoire populaire se trouvent dans une situation fluctuante, entre l'absence et la présence. Dans leur vieille demeure on attend inlassablement leur retour :

«Los que desaparecen se borran cierto tiempo de su casa, de las cosas, de sus gentes, de los amigos, de los espejos solitarios. Se sabe de ellos porque pueden regresar, posibilidad que los mantiene de cuerpo ausente entre todas las cosas. Están ausentes y presentes. No están y están por no ser extraños a los que con ellos vivieron y se quedaron. Su presencia es un recordado vacío de su forma entre los contemporáneos. Si regresan ese vacío se llena de nuevo, se llena con ellos que son ellos y sus recuerdos. » (p. 25-26).

8 Hormis le «corredorcito " qui joue un rôle essentiel dans cette première partie, au moins deux autres espaces ont une fonction déterminante : le "charco del limosnero » («la flaque du mendiant») et le cirque.

9 Par des raisons d'espaces nous n'avons pas la possibilité de nous étendre sur cette partie. Disons cependant que tout se passe ici dans la dimension imaginaire du "Alhajadito ", mais dans cet espace onirique, les voix de plusieurs personnages s'emboîtent : El Párroco, Bernardo el Niño, Joper el Niño, Luis Pino, Pablo Figo, Afre. 
Le « corredorcito » est le point de départ matériel des divagations oniriques du protagoniste, à la recherche de réponses aux disparitions mystérieuses, d'explications à ce qui est arrivé dans cette maison délabrée et habitée par des serviteurs indiens ${ }^{10}$. Mais si dans un premier temps le petit couloir apparaît aux yeux du protagoniste comme inhabité, abandonné, progressivement il se peuple de petites créatures (d'insectes), de rêves et de voix ${ }^{11}$.

Une voix narrative qui se présente au début du roman comme un narrateur extradiégétique, partant d'une perspective externe, introduit le personnage et son espace, le " corredorcito ». La séquence décrit le protagoniste enfant en train de mâcher un bout de canne à sucre dans le petit couloir :

«Bigotes de miel de caña de azúcar. Por las comisuras le bajaban como puntas de bigotes chinos, tostaditos, cosquillosos, dulces al lamerlos con lengua de gato. Tenía que defenderse de las moscas a manotazos. Defender sus bigotes. (...) La casa tenía olvidado muy a trasmano un trecho de corredor. No daba a ninguna puerta, a ninguna ventana. Simplemente a la espalda de una pared lisa que lo separaba de unos cuartos para aparejos y otros tropiezos. » (p. 9)

Cette focalisation de départ se modifiera très rapidement à un moment significatif du texte, lorsque le narrateur fait connaître le rapport entre personnage et espace : «El corredorcito, su corredorcito... [...] El corredor aquél. Aquel $s u$

10 Cette recherche d'une explication du passé et donc de ses propres origines est comprise par l'un des personnages clés du roman, Mendiverzúa, lorsqu'il dit : "i ; Pues si que tiene gracia, yo, tamaño lobo de mar y ustecito tamaño preguntón en busca de la radiz de todo lo que pasó aquí en su casa! » (p. 74).

11 La surprise face à cet espace habité contrairement aux apparences laisse le protagoniste perplexe : «¿Cómo ? ... ¿Había tanto ser vivo en aquel trecho de corredor del que él se consideraba único habitante ? [...] ¡Y él que creía estar solo y ser el único dueño del corredorcito!" (p. 14). Finalement le couloir sera occupé par un personnage d'apparence grotesque, Surilo, « el contrahecho pescador que preparaba la carnada para los anzuelos. » (p. 48). L'abandon du "corredorcito" qui se produit à la fin de la Première Partie, symbolise le passage de l'enfance à l'adolescence. 
corredorcito... $»^{12}$. Le possessif et le diminutif introduisent ici l'affectivité et par conséquent la dimension subjective qui vient se superposer et traversera, en la transformant, la vision externe que le narrateur avait présentée de cet espace au début du roman. Dans la mesure où ces marques de subjectivité se multiplient, une profonde complicité et identification entre personnage et narrateur s'établit. Dès lors, le passage intermittent de la focalisation externe à la focalisation interne s'accomplit, créant de cette manière un jeu énonciatif ambigu, car les deux voix s'amalgament, s'exprimant simultanément. Ainsi, lorsque protagoniste et narrateur découvrent des traces inusitées dans leur espace, ils réagissent : "¿Quién tiznó la pared ? ¿Quién vino anoche al corredorcito ? Ayer no estaba aquella como rajadura. ¿Quién ? ¿Quién ?» (p. 10-11). L'énonciation peut venir de l'une ou l'autre entités, ou des deux en même temps. On comprend ainsi que l'univers narratif sera une projection de la conscience du personnage. Mais voilà que c'est dans cette intériorité que le jeu de l'énonciation se complique et s'enrichit car la conscience du «Alhajadito » n'est pas unidimensionnelle. Elle ne se compose pas seulement de souvenirs personnels, mais également des voix de la tradition populaire qui détermineront son propre imaginaire.

Le protagoniste fait du «petit couloir » son domaine, il s'approprie l'espace au moyen de l'imagination, en nommant les objets qui y sont présents, en régnant sur la vie ou la mort des petites créatures (insectes), en simulant des tremblements de terre par des coups assenés sur les colonnes ${ }^{13}$. Mais c'est surtout l'acte de nommer son domaine, à voix haute, avec ses propres mots, ce qui lui donne la sensation de l'intégrer pleinement à son monde intérieur ${ }^{14}$. En faisant de cet espace

12 C'est l'auteur qui souligne, p. 9-10.

13 La perspective de la voix enfantine est privilégiée dans cet acte d'appropiation, par l'imaginaire, de la réalité : "Estaba inmóvil, furioso devorador de cañas dulces, fabricante de pequeños terremotos, dueño de un corredorcito habitado, como las casas, por muchos seres invisibles, y de un tesoro de monedas sucias de ceniza. " (p. 15)

14 Le narrateur qui raconte en intériorisant la voix de l'enfant dit : "Oírse hablar le dio la mayor seguridad. Llamar al pilar, pilar, al ladrillo, ladrillo, pared a la pared, le creaba una superioridad, una 
son domaine, "l'Alhajadito » est alors dans la condition de libérer son imaginaire, de faire émerger son univers de rêves et de fabulation. Dans cette logique de lecture, le « corredorcito » peut s'interpréter comme l'allégorie de cette conscience enfantine qui, elle aussi, en cherchant des réponses et des explications aux énigmes qui l'entourent, se remplit. Elle se transforme donc en un espace occupé de voix.

Le "petit couloir » est l'axe central à partir duquel toutes les histoires qui composent le roman s'enchaînent, convergent et se déploient. Quelles sont ces autres voix qui s'enchâssent dans la conscience du protagoniste et que racontent-elles ? Ces voix multiples qui se reconstituent dans le monde intérieur du protagoniste, proviennent de personnages de l'univers populaire que "l'Alhajadito » côtoie quotidiennement, surtout les pêcheurs et les servantes. Mais aussi de membres de sa famille. Ainsi, l'enfant reconstruit dans sa conscience la voix de son grand-père qui raconte les histoires de ceux qui se sont noyés dans la «Flaque du Mendiant ». Ce sont des histoires qui se transmettent de génération en génération, jusqu'au moment où elles prennent place dans la fantaisie de l'enfant ${ }^{15}$. Dans ce processus de « convocation » de voix, à l'instar du « corredorcito », la «Flaque » apparaît sous une dimension qui transcende le «réel ». Et c'est la multiplication des instances énonciatives qui ouvrira cette dimension. En effet, «La Flaque » n'est plus seulement la lagune d'eau sale - de là le nom qu'elle porte selon l'une des explications que propose le texte - où les pêcheurs tirent leurs moyens de subsistance. Mais elle est aussi, comme le raconte le personnage du grand-père, un

majestad. Un esfuerzo hablar a solas, conversar con las materias sordas, mudas, insensibles. Pero cuánta autoridad tiene la palabra. » (p. 13)

15 L'une des voix des pêcheurs occupe plus fréquemment son imaginaire, c'est celle de Mendiverzúa ( $c f$. supra). Certaines constructions lingüistiques permettent d'établir la nature orale de ces histoires: "Y de ese frasear enigmático no pasaban los pescadores mientras remendaban las redes. Así lo oyeron decir a la gente de antes y lo repetían como él se quedó repitiéndolo, camino al corredorcito, al desaparecer los pescadores. » (p. 16) ; «Una frase de los pescadores le revoloteaba en la boca y hasta hizo el distraído ademán de espantársela, como a una mosca... » (p. 25) ; «Y palabra el viejo, palabra el niño, llegaron al corredorcito. " (p. 78). 
cimetière lacustre («Un camposanto sin cruces. », p. 19), le lieu où sont submergés - noyés ?, disparus ?, morts ? - les ancêtres de l'enfant. Or la lagune est également - d'après le plus vieux des pêcheurs, Mendiverzúa - l'endroit dans lequel l'un des ancêtres du protagoniste, nommé " Azacuán »16 a disparu après son mariage avec un cerf-volant, la métaphore de son aimée Indiga : "Parece cuento y no es cuento. », dit Mendiverzúa. Indiga disparaît à son tour et « l'Azacuán » se met à sa recherche :

"Vestido con la cogulla bordada de grillos, pedía limosna por los caminos y buscándola en los espejos, desapareció una noche en las aguas del Charco que desde entonces se llama del limosnero. » (p. 83) $)^{17}$

Grâce à la voix de Mendiverzúa, ainsi qu'à celles du grandpère et des autres pêcheurs qui résonnent dans la conscience du « Alhajadito », la « Flaque » devient un espace générateur de fantaisies et de contes merveilleux. A travers la voix du vieux pêcheur, reconstruite dans la mémoire du personnage enfantin, d'autres voix sont rapportées, en suivant un jeu complexe qui comprend discours direct, indirect ou indirect libre. Cette dynamique énonciative a pour résultat une polyphonie narrative où les voix alternent et se superposent, de telle sorte qu'elles s'enchaînent à des degrés divers. Voyons l'une de ces chaînes possibles: la conscience du "Alhajadito » rapporte la voix de Mendiverzúa qui, à son tour, rapporte celles des personnages qui participent dans les fantaisies et les contes merveilleux (celui du cerf-volant ou celui de la Cloche du Mauvais Larron). Le modèle utilisé est par conséquent celui de la tradition orale, dans laquelle les voix s'englobent les unes et les autres, de telle manière que

16 Comme cela est assez fréquent dans l'oeuvre romanesque d'Asturias, l'explication du nom que portent les personnages a une dimension métaphorique : "Le llamaban el Azacuán por su traje de color negro amarillento, por su pelo ralo pegado a la redondez de la cabeza pequeña como peluquín de pluma y porque, como los azacuanes, emigraba en invierno de una habitación a otra de la casa llevando su cama, su mesa, su silla, sus libros... " (p. 42). Azacuán : terme utilisé au Guatemala pour désigner un oiseau migrateur. On les trouve dans la région du Petén, au Nord du pays.

17 C'est l'auteur qui souligne. 
l'individu parlant finit par s'effacer au profit d'une voix collective. Et c'est justement cette construction d'une voix collective le projet qu'Asturias a poursuivi dans son œuvre romanesque - Hombres de Maíz (1949), Mulata de tal (1963).

Hormis la voix de Mendiverzúa et du grand-père, mort durant un jour de pluie ${ }^{18}$, celles des servantes viennent s'ajouter à cette polyphonie, contribuant à créer une atmosphère où la réalité devient floue. Ne trouvant aucune réponse à l'absence du grand-père, l'enfant interroge l'une des servantes qui répond :

«-Por el señor pregunta...? ¡Desapareció...! ¡En esta casa todos desaparecen!» (p. 31)

Pêcheurs, grand-père, servantes sont des voix qui résonnent dans l'imaginaire du protagoniste, et c'est à partir de cette intériorisation des voix que nous découvrons son univers. On a donc tout un enchaînement de microtextes qui émergent de cette conscience enfantine laquelle, dans la Première Partie du roman, s'exprime depuis le "corredorcito ». Cette situation spatiale est essentielle car c'est elle qui donne la clé de l'enchâssement des voix dans celle du protagoniste. L'ensemble de ce qui est raconté se déroule alors que l'enfant se trouve toujours dans le petit couloir ${ }^{19}$. Le " corredorcito » fonctionne comme un point de mire; c'est un point d'observation et de reconstruction de l'univers mystérieux, c'est de là que le protagoniste voit, interroge et imagine son monde. Car personne ne semble vouloir lui donner d'explication à son

18 L'épisode a lieu dans le chapitre VIII. Comme cela est le cas pour la plupart des faits qui sont relatés dans le roman et suivant la logique de la stratégie narrative, celui-ci apparaît imprécis, vague dans la conscience du protagoniste : " ¿Se le murió en el corredor? ¿Ya estaba muerto cuando lo recogió ? ¿Murió ahogado? ¿Murió de frío ? ¿Murió de viejo... ? " (p. 30)

19 Quelques constructions lingüistiques nous le confirment: «Estaba como siempre inmóvil en el corredorcito » (p. 21); «En su corredorcito, allí donde estaba. » (p. 26). Les constructions de ce type servent de repères spatiaux pour le lecteur qui déduit que l'univers raconté et les voix des personnages émergent de la conscience enfantine qui occupe le petit couloir. 
égard : "El Alhajadito imaginaba lo que creía que las criadas le callaban de sus antepasados... » (p. 43) ${ }^{20}$.

L'énigme de ces questions sans réponses s'éclaircit, partiellement, dans la Troisième Partie du livre. En effet, si dans la Première Partie la voix qui organise la narration est celle du protagoniste enfant, dans la dernière ce n'est plus l'enfant mais l'adulte qui conduit le récit. La voix s'est donc dédoublée pour s'exprimer à partir d'une nouvelle perspective. On comprend alors que le roman dans son ensemble - et tout particulièrement la Première Partie - est structuré par une série d'évocations oniriques que l'Alhajadito élabore pour essayer de clarifier ce que les adultes ne veulent ou ne peuvent lui expliquer à propos de son origine : une naissance illégitime et une étrange solidarité entre deux sœurs qui n'ont jamais dévoilé laquelle des deux femmes est la mère de l'enfant ${ }^{21}$. De cette énigme irrésolue surgit dans la conscience enfantine la nécessité d'une explication qu'on ne lui fournit pas. La réponse qu'il trouve c'est l'imaginaire, l'invention.

A partir de la perspective de l'adulte, le narrateur construit une autre lecture de son enfance en faisant appel à ses souvenirs. Le résultat de cette nouvelle lecture est une réinterprétation d'éléments imaginaires déjà présents dans la Première Partie. Les épisodes reconstruits présentent l'enfant et ses deux mères dans une grande maison qui progressivement tombe en ruine, car la pauvreté menace les trois personnages. Les visites régulières des membres de l'Institut de la Charité, que l'enfant perçoit de manière vague et menaçante, marquent l'intensification des pénuries économiques, jusqu'au jour où ils ne reviennent plus : l'Institut ne prête pas son concours aux familles avec des enfants illégi-

20 D'autres passages confirment cette situation énigmatique : «el misterio que rodeaba aquel mundo de su infancia" (p. 20). "Ríos secretos que alimentaban su secreto, su gran misterio ». (p. 21)

21 Dans la dernière partie, le narrateur adulte exprime : « No conocí a mi padre y mi madre fueron dos mujeres tan suaves que su memoria me entristece, deprimiéndome igual que si me pusiese a pensar en cosas tristes. " (p. 121) 
times $^{22}$. C'est alors que les usuriers font des apparitions régulières :

«Una de mis mamás, aquella mañana, vino con el cofrecito de sus alhajas a donde estaba el visitante, bajo la acción de una fuerza extraña... » (p. 126)

Il est impossible de ne pas établir un lien entre cet épisode des joyaux et la famille des «Alhajaditos » de la Première Partie. De plus, les personnages des usuriers se voient attribuer certains signes qui les rapprochent étrangement des " Alhajados », en particulier les habits noirs qu'ils portent et l'attente mystérieuse de leur retour. Une interférence se noue donc entre ces personnages. C'est ainsi que cette nouvelle lecture que le narrateur adulte élabore de son monde, soulève l'hypothèse selon laquelle, d'une part, tout l'univers de la Première Partie est une invention de l'enfant dans sa recherche de réponses à son origine, et de l'autre, la voix narrative du début du roman, qui fait alterner la focalisation externe et interne, est celle de l'adulte qui se voit en tant qu'enfant et rapporte son imaginaire et ses mots. Des éléments essentiels qui composaient l'univers imaginaire de l'enfant dans la Première Partie se retrouvent dans cette partie mais sous une autre apparence : la maison mystérieuse des " Alhajados » où l'on attendait leur retour est maintenant une vieille demeure silencieuse, obscure, qui se vide progressivement avec les visites des usuriers ; la « Flaque » est maintenant un étang dans un jardin. Le monde populaire des pêcheurs et de Mendiverzúa entre en parallélisme avec le monde du jardinier Eduviges dont le fils est un enfant aveugle. Ce personnage, non moins énigmatique, sera, en un premier temps, le compagnon aimé de l'Alhajadito, mais plus tard, lorsqu'il lui dévoilera le secret de sa naissance, il le haïra. Or ce secret dévoilé, comme c'est le cas pour tous les faits relatés dans le roman, est le résultat du dire, de ce que les voix expriment et de ce l'on écoute, de l'oralité. Le petit aveugle confesse à « l'Alhajadito » :

22 «Las oí decir - dit-il - que el Instituto no auxiliaba familias con hijos ilegítimos. » (p. 125) 
«No sé si contarte lo que oí decir el otro día. Conversaban tus mamás con mi papá. [...] Tú vivías, según ellas, en una casa muy grande, muy grande, muy grande, rodeado de criados con trenzas, no lejos de un lago, entre pescadores, y jugabas a pirata en un corredorcito. De esa casa donde, ¡Dios sea con nosotros!, se rendía culto al Mal Ladrón, desaparecían los señores que, como tú, vestían siempre de negro. [...] Uno de aquellos hombres vestidos de negro (...) sedujo a la más hermosa de dos hermanas gemelas. Le robó la honra y desapareció. Temerosas de lo que tu abuelo haría, escaparon ambas y al volver a la casa paterna, ya venías tú; pero ninguna de las dos confesó a su padre, quién de ellas era tu mamá. ¡Un hijo de dos madres deshonradas! » (p. 125)

Univers du dire, de voix, est donc celui de El Alhajadito. Des voix qui résonnent en permanence et à partir desquelles se reconstruit et se déforme un monde qui reste insaisissable dans la conscience du protagoniste. Parmi cette multiplicité de voix, celle de la tradition orale n'est pas absente dans cette Troisième Partie.

Si dans la Première Partie, le personnage de Mendiverzúa introduit des histoires de nature fantastique et poétique (Cf. le mariage du «Azacuán » avec le cerf-volant, la Cloche du Mauvais Larron, le cimetière des pirates dans la « Flaque du Mendiant $»^{23}$ ), ici c'est Eduvijes, le jardinier, qui accomplit cette fonction. Dans le chapitre V de cette partie, cinq contes que le jardinier racontait à l'enfant s'insèrent dans le texte en une évidente " mise en abîme ". Le narrateur adulte, en sollicitant ses souvenirs, rapporte la voix du conteur : «Eduvijes (...) - dit-il - me contait les « Cuentos del Cuyito ». » (p. 130). Les cinq contes titrés : "Clarín Clarinero », "La Palomita Verde », " Corazón de Aguacate », «Zopilotes blancos » et "La Maquinita de hablar» sont bâtis en suivant le modèle des contes enfantins de la tradition orale populaire guatémaltèque. Avec des personnages du monde Naturel - faune et flore -, l'humour et le surnaturel, l'animisme, la recherche de

23 C'est cette histoire qui génère la Deuxième Partie, le rêve du bateau fantôme. Toute cette partie est par conséquent une "mise en abîme ", c'est pourquoi il présente une " autonomie relative ». 
rythmes et de rimes et les sentences morales, ces microtextes présentent à leur tour une grande autonomie. Il s'agit d'histoires complètes, achevées que le personnage d'Eduviges, par l'intermédiaire de "l'Alhajadito " adulte, insère dans le macrotexte du roman. Ces histoires démontrent dans quelle mesure, en étant présentes dans l'imaginaire du protagoniste, elles finissent par structurer sa vision du monde. C'est pourquoi si le secret de son existence lui échappe, l'Alhajadito peut s'approprier ce mystère à travers l'acte d'imaginer. Par cet acte il fait sienne la « réalité » car il agit sur elle.

A la fin du roman, force est d'accepter que la problématique d'identité de l'enfant n'est pas résolue, et qu'elle n'est peut-être qu'un prétexte pour déclencher le jeu de l'imagination et évoquer sa répercussion sur le réel. Le roman reste toujours dans les brumes de la réalité-rêves : "Adivinaba lo que había pasado o lo estaba inventando » (p. 21) se demande "l'Alhajadito ». La réalité apparaît comme une hallucination pour le personnage, un jeu d'illusions :

«De modo que mis mamás... De modo que el corredorcito... los criados trenzudos... el Mal Ladrón, el circo... Ana Tabarini... el Negro Pispís... el Párroco... el carruaje del Instituto de Caridad, todo había sido un sueño... [...] Me persigné... ¡Por la señal de la Cruz!, iba a decir, pero dije ¡Por la señal de los sueños...! » (p. 161)

$$
\text { *** } *
$$

Dans la logique du roman, l'univers du Alhajadito reste comme une possibilité multiple, car elle est racontée, évoquée, suggérée par une pluralité de voix ( Cada quien en este mundo se fabrica su verdad. » p. 50).

Ce phénomène polyphonique révèle la concrétisation du projet esthétique asturien : l'élaboration d'une voix collective qui s'efforce de traduire son univers culturel. Vu sous cet angle, l'apparente structure fragmentée, dispersée du roman ne l'est plus, car l'hétérogénéité des voix apparaît comme la solution narrative nécessaire pour traduire un univers culturel profondément marqué par l'oralité. 
Le jeu entre oscillation et dédoublement permet la création de l'espace-imaginaire, de l'espace culturel : là où convergent le monde de chacun et le Monde ; l'histoire de l'individu et celle de sa "tribu », comme dirait le "Gran Lengua ». Un espace qui ne peut être reconstruit que par l'appropriation, l'assimilation et la réinvention de la parole du groupe. Dans son étonnement, "l'Alhajadito " nous livre peut-être la clé de son univers (et de la réalité) :

« ¡Cómo podía ser que tanta realidad desembocara en tanto sueño!»(p. 86). 